\title{
Plataformas nanoestructuradas de plata para identificación cualitativa de Escherichia coli mediante espectroscopia Raman intensificada por efecto de superficie "prueba de concepto del sistema"
}

\section{Silver nanostructured platforms for detecting Escherichia coli through Raman scattering spectroscopy "Proof of concept"}

\author{
John Jairo Castillo-León \\ Ph.D. Química \\ Universidad Industrial de Santander \\ Bucaramanga, Colombia \\ jcasleon@uis.edu.co
}

\author{
Bladimiro Rincón-Orozco \\ Ph.D. Ciencias Naturales \\ Universidad Industrial de Santander \\ Bucaramanga, Colombia \\ blrincon@uis.edu.co \\ Rafael Cabanzo-Hernández \\ M.Sc. Ciencias Naturales \\ Universidad Industrial de Santander \\ Bucaramanga, Colombia \\ rcabanzo@uis.edu.co
}

Resumen- La espectroscopia Raman intensificada por efecto de superficie (SERS) de materiales nanoestructurados es una técnica ultrasensible que permite alcanzar niveles de detección extremadamente bajos en la determinación de diferentes analitos. En este estudio se logró identificar cualitativamente la bacteria $E$. coli, utilizando plataformas nanoestructuradas de plata, mediante la técnica SERS. Las plataformas nanoestructuradas de plata (AgNP) fueron fabricadas por la técnica de grabado mediante iones reactivos con posterior deposición de una capa metálica de plata. Las bacterias fueron preparadas en medio Luria-Bertani (LB) a $37^{\circ} \mathrm{C}$ por 4 horas. De este cultivo de bacterias 5 uL fueron depositados sobre la superficie de las AgNP, dejándose secar por $\mathbf{3 0}$ min. Posteriormente, el sistema AgNP-bacteria fue analizado mediante espectroscopia Raman. La identificación de la banda Raman a $731 \mathrm{~nm}$ típica de la $E$. coli sobre las AgNP permitió identificar cualitativamente la bacteria, lo que brinda la posibilidad de utilizar las plataformas como potenciales biosensores ultrasensibles de bacterias nosocomiales en ambientes intrahospitalarios.

Palabras clave- Plataformas nanoestructuradas de plata, SERS, bacteria, nanotecnología.

Abstract- Surface enhanced Raman spectroscopy (SERS) of nanostructured materials is a powerful technique that allows to reach ultrahigh levels of detection of several analytes. In the present study it was possible to identify the bacteria $E$. coli by using a novel nanostructured platform based on silver-capped nanopillars (AgNP) and SERS technique. AgNPs were fabricated by ion reactive etching and deposition of silver layers. The bacteria culture were prepared in Luria-Bertani (LB) medium at $37^{\circ}$ by 4 hours. $5 \mu \mathrm{L}$ of the bacteria were deposited on top of the surface of AgNPs and let it dry for $\mathbf{3 0}$ minutes. Subsequently the AgNP-bacteria system was analysed by Raman spectroscopy. A typical band of $E$. coli at $\mathbf{7 3 1}$ cm-1 was identified and this Raman vibration was used as a marker peak to detect the bacteria. Finally the novel AgNP could be used as a potential biosensor to detect nosocomial bacteria in intra-hospital environments.

Keywords- Silver nanostructured platforms, SERS, bacteria, nanotechnology.

\section{INTRODUCCIÓN}

Una de las motivaciones para la fabricación de sustratos nanoestructurados de metales nobles soportados sobre superficies sólidas es la dificultad que presentan algunos coloides de nanopartículas (NP) de oro y plata en la obtención de espectros SERS [1]. Esta dificultad radica principalmente en la tendencia a la aglomeración de las NP metálicas luego de su interacción con el 
analito, lo que conlleva la obtención de espectros SERS poco reproducibles, con presencia de ruido y difíciles de interpretar.

Existen diferentes técnicas químicas y físicas para la fabricación de nanoestructuras metálicas soportadas sobre superficies sólidas. Entre las más utilizadas se encuentran: evaporación al vacío, deposición electroquímica, pulverización catódica (sputtering) y plateo electroquímico [2-4]. Otra alternativa la constituyen los métodos basados en la nanoimpresión de metales sobre superficies de silicio, técnica conocida como nanolitografía. Recientemente el grupo de Boisen y colaboradores desarrolló una metodología para la fabricación de plataformas de sustratos nanoestructurados de oro y plata sobre nanopilares de silicio, utilizando la técnica de grabado mediante iones reactivos (RIE) [5]. Estos nanopilares de oro y plata (AuNP o AgNP) han sido utilizados con gran éxito para la detección de biomoléculas como la vasopresina y el ácido fólico [6]. El principio por el cual ocurre el aumento en la intensificación de las señales Raman de diferentes tipos de biomoléculas se ilustra en le Fig. 1C. Básicamente luego de la evaporación del solvente las moléculas del analito se depositan en el medio de las cabezas del metal (Ag) de las plataformas alineándose de tal forma que dan lugar a la creación de "hot spots" con el consecuente aumento en el campo electromagnético y la intensificación de la señal Raman.

La aparición de microorganismos multiresistentes ha llevado no solo al desarrollo de nuevos fármacos que los combatan, sino a la creación de herramientas y mecanismos que permitan su detección temprana, ya que muchas de estas cepas son altamente virulentas y la gravedad de las afecciones que causan depende en gran medida del tiempo que se tarda en realizar un diagnóstico acertado [7]. En la última década, con todo el desarrollo en el área de la nanotecnología, se ha logrado obtener notables resultados en la detección de agentes patógenos empleando técnicas espectroscópicas, siendo este un método versátil y robusto para el tratamiento de diferentes matrices [8].

Una de las investigaciones más relevantes fue propuesta en 2014 por Zhou y cols [9] en la que emplean SERS para la detección de la cepa E. coli DMS 498/1116/5695 y S. epidermidis 61741 en muestras de agua potable, usando NP de plata; estas AgNPs fueron sintetizadas previamente y se adhirieron a la pared celular de la bacteria permitiendo la intensificación de las señales Raman. La intensidad de la señal Raman depende en gran medida del potencial de las NP y de la membrana bacteriana que interaccionan electrostáticamente. El método implementado permitió el análisis de muestras (AgNPs-Bacteria) en un volumen de 1 $\mathrm{mL}$, tiempo de análisis de 10 minutos, alcanzando un límite de detección de $2.5 \times 10^{2}$ células $/ \mathrm{mL}$. Este estudio demostró la selectividad y sensibilidad de la técnica SERS para la detección de estas dos bacterias, el cual puede ser extendido al desarrollo de otros biosensores.

En este mismo año, Graham y cols desarrollaron un método para la determinación y cuantificación simultánea de tres bacterias patógenas causantes de meningitis empleando SERS. El ensayo cuantitativo para la detección de Neisseria meningitidis, Streptococcus pneumoniae y Haemophilus influenzae requirió la hibridación simultánea de dos hebras de ADN complementario (una que contenía el cromóforo activo SERS) a la secuencia objetivo, seguida de la digestión de una cadena doble de ADN por acción de la $\lambda$-exonucleasa para la posterior detección SERS del producto de la digestión para cada patógeno de forma individual. Se obtuvieron resultados consistentes para la detección de meningitis bacteriana causada por estás tres bacterias, con límites de detección para S. pneumoniae fue igual a $99.5 \mathrm{pM}$; N. meningitiis fue igual a 45.3 pM y para $H$. influenzae fue igual a $21.7 \mathrm{pM}$. Pese a que la detección de estas bacterias en una mezcla es compleja, Ser recomienda realizar estudios de las características espectrales de las señales SERS combinada con una regresión de mínimos cuadrados parciales [10].

Este trabajo presenta la identificación de la bacteria $E$. coli a través de la espectroscopia SERS mediante el uso de plataformas basadas en nanoestructuras de plata. Para conseguir esta identificación se estudió el espectro SERS de la E. coli mediante el análisis y asignación de las principales vibraciones características. Esta información es fundamental para identificar la huella digital de $E$. coli convirtiendo a las AgNP en potenciales sistemas de detección para análisis rápido y confiable de poblaciones de bacterias nosocomiales presentes en ambientes intrahospitalarios. Esta es una importante problemática que afecta al año 
aproximadamente 7 millones de pacientes en países desarrollados [11]. La estadística en países en vías de desarrollo estima una prevalencia de casos que varía entre $5 \%$ y $19 \%$ de los pacientes hospitalizados, en Colombia, el Ministerio de Salud reportó entre los años 1996 al 2000 un porcentaje de infecciones nosocomiales entre el 2,31\% al 2,55 \% del total de pacientes hospitalizados [12].

\section{METODOLOGÍA}

\subsection{Fabricación de las plataformas nanoestructuradas de plata}

Las AgNP fueron fabricadas en el departamento de Micro y Nanotecnología de la Universidad Técnica de Dinamarca [6]. Inicialmente un cd de silicio fue sometido a un proceso de grabado mediante iones reactivos (RIE, por sus siglas en inglés) para la obtención de pilares de un tamaño aproximado de $200 \mathrm{~nm}$. Posteriormente, mediante un tratamiento con plasma se removieron los subproductos del proceso RIE y finalmente se depositó una capa metálica de plata por medio de deposición de vapor por haz de electrones. Las dimensiones de los AgNP fueron de $600 \mathrm{~nm}$ de altura y $50 \mathrm{~nm}$ de ancho como se aprecia en la Fig. 1. Las AgNP fueron almacenadas en un desecador para evitar problemas de oxidación.

\subsection{Preparación de los cultivos de E. coli}

Las bacterias se prepararon como se describe brevemente: E. coli cepa top10F (Invitrogen) se cultivó en medio Luria Bertini a $37{ }^{\circ} \mathrm{C}$ hasta obtener una D.O. de 0.5 a $600 \mathrm{~nm}$. Posteriormente, un mililitro de bacterias fue mezclada con $3 \mathrm{ml}$ de Buffer Salino Fosfato $(\mathrm{pH} 7,4)$. Subsecuentemente, se realizaron tres lavados por centrifugación de $10 \mathrm{~min}$ a $5800 \mathrm{rpm}$. El pellet obtenido se volvió a suspender en $500 \mathrm{~mL}$ de Buffer Salino Fosfato quedando listo para la medición por SERS.

\subsection{Obtención de espectros SERS de E. coli}

Se depositaron $10 \mathrm{~mL}$ de solución con la bacteria, donde la solución de $E$. coli fue esparcida sobre el área total del chip de AgNP (5x5 $\left.\mathrm{mm}^{2}\right)$ para asegurar homogenización de la muestra sobre la superficie y lograr el tiempo suficiente de adsorción e interacción de la bacteria con la plata, y evitar problemas de desorción. Luego, se dejó evaporar por aproximadamente 30 minutos a temperatura ambiente. Antes de la toma del espectro SERS las AgNP fueron cui- dadosamente lavadas con un buffer fosfato 10 $\mathrm{mM} \mathrm{pH} 7.0$ para remover las bacterias que no se adsorbieron. La señal del espectro Raman fue medida en un área aproximada de 0.05 $\mathrm{mm}^{2}$ utilizando un láser de $785 \mathrm{~nm}$ a una potencia de $0.5 \mathrm{~mW}$. Todas las mediciones SERS se realizaron en un microscopio Raman Horiba Scientific LabRam HR Evolution en un rango de números de onda entre $50-4000 \mathrm{~cm}^{-1}$.

\section{RESULTADOS}

\subsection{Espectro SERS del ácido fólico}

La espectroscopia SERS es una técnica que al combinar la espectroscopia láser con las propiedades ópticas de las nanoestructuras metálicas permite obtener un incremento de la señal Raman proveniente de diferentes tipos de biomoléculas y microorganismos [13]. A pesar de que existen numerosos reportes sobre la determinación de bacterias utilizando la técnica SERS mediante el uso de nanopartículas de metales nobles en solución [14], en la literatura revisada no encontramos publicaciones que reporten el uso de plataformas nanoestructuradas sólidas de plata para detección de E. coli. La determinación de bacterias patógenas es de vital importancia para la salud pública, debido a que cada día es necesaria la búsqueda de nuevos sistemas de detección con diagnósticos oportunos que permitan obtener una respuesta rápida y confiable de un analito en particular.

Con el propósito de evaluar el funcionamiento de las AgNP preparadas en este trabajo, se determinó el espectro SERS de un compuesto conocido y caracterizado anteriormente, este compuesto fue el ácido fólico (AF). En la Fig. 2 se puede apreciar el espectro SERS del AF depositado sobre la superficie de la AgNP y como se observa la mayoría de señales Raman coinciden con las señales obtenidas en trabajos anteriores [5]. Típicamente las vibraciones representativas del AF se encuentran en 1569, 1513, 962 y $684 \mathrm{~cm}^{-1}$ las cuales corresponden a modos de vibración de tipo balanceo, estiramientos, y aleteo de los grupos $\mathrm{NH}_{2}, \mathrm{C}-\mathrm{N}$, $\mathrm{CH}_{2}$ y O-H de la estructura del AF, respectivamente. La Fig. 3B muestra el espectro SERS en ausencia del AF

\section{2 Espectro SERS de E. coli}

Posteriormente y luego de comprobar el funcionamiento de las AgNP se adquirió el espectro 
SERS de una solución conteniendo la bacteria $E$. coli. En la Fig. 3A se indican las señales Raman de las vibraciones más probables y que están relacionadas con la presencia de la bacteria. Con el objetivo de determinar la homogeneidad de la superficie de AgNP se midió un espectro Raman control en ausencia de muestra para comprobar que las señales son exclusivamente provenientes de la bacteria Fig.3B.

La Fig. $3 \mathrm{C}$ muestra la intensidad de la señal Raman a $731 \mathrm{~cm}^{-1}$ en ausencia y en presencia de la solución de E. coli. De lo anterior se puede deducir que la superficie del sustrato SERS es totalmente homogénea y la única señal Raman que se presenta es debido al silicio que hace parte de la estructura de las AgNP. Por lo tanto se puede confirmar que las señales Raman son debidas a los diferentes modos de vibración presentados por los grupos funcionales que hacen parte de la estructura molecular de la bacteria. Un análisis más detallado de los principales modos de vibración se puede apreciar en la tabla I. A modo de experimento control se aprecia el espectro SERS del medio de cultivo sobre los nanopilares de plata Fig.3D.

\subsection{Modos de vibración representativos de E. coli}

De especial interés se encuentra el modo de vibración del anillo de la glucosa a $731 \mathrm{~cm}^{-1}$ que pertenece a los peptidoglucanos de la pared celular bacteriana, los cuales son ricos en $\mathrm{N}$-acetil-D-glucosamina (NAG). En estudios similares este modo de vibración es utilizado como huella digital para la determinación e identificación de E. coli [ 7,14]. Otros modos de vibración importantes presentes en el espectro SERS están relacionados con la presencia de lípidos y ácidos nucleicos presentes en la estructura de E. coli [15]. Por ejemplo la señal
Raman a $1451 \mathrm{~cm}^{-1}$ está relacionada con el modo de vibración tipo estiramiento simétrico del grupo $\mathrm{CH}_{2}$ presente en las proteínas. Por otro lado, la señal a $1608 \mathrm{~cm}^{-1}$ indica un modo de vibración tipo estiramiento de los anillos de adenina y guanina presentes en los ácidos nucleicos. La señal a 1000 $\mathrm{cm}^{-1}$ corresponde a un modo de vibración de tipo estiramiento atribuido a la difenilalanina presente en la parte hidrofóbica de la membrana celular.

El incremento de las señales Raman de la solución de E. coli se puede explicar mediante el siguiente mecanismo. En primer lugar, luego de la evaporación del agua, que hace parte de la solución de las bacterias, estas se acomodan en los espacios inter-nanopilares de Ag. Posteriormente, producto de la presencia de las bacterias y su interacción con el láser incidente, los pilares se alinean entre sí creando de este modo hot spots (zonas de aumento de campo electromagnético) que incrementan la intensidad de la señal Raman. Este mismo efecto ha sido utilizado para explicar el efecto SERS en otros estudios, utilizando sustratos diferentes [3, 7, 16-19].

Finalmente, con el concepto de prueba del sistema se evidencia el uso potencial de las AgNP como posibles biosensores espectroscópicos para la detección cualitativa de bacterias presentes en ambientes hospitalarios, mediante la interpretación de su espectro Raman a través de la identificación de su huella digital a $731 \mathrm{~cm}^{-1}$ principalmente.

\section{CONCLUSIONES}

Se logró la identificación cualitativa de una muestra de la bacteria E. coli por medio del efecto intensificado por superficie de la señal Raman sobre nanoplataformas de plata. El pico marcador Raman se identificó en $731 \mathrm{~cm}^{-1}$ señal perteneciente

Tabla I

ASIGNACIÓN DE LOS MODOS DE VIBRACIÓN RAMAN DE LA BACTERIA E. COLI

\begin{tabular}{|l|l|}
\hline \multicolumn{1}{|c|}{ Señal Raman $\left(\mathbf{c m}^{-1}\right)$} & \multicolumn{1}{|c|}{ Modo de vibración } \\
\hline 1608 & Estiramiento anillo de adenina y guanina \\
\hline 1567 & Tijereteo de $-\mathrm{NH}_{2}$ de las proteínas \\
\hline 1451 & Deformación de $-\mathrm{CH}_{2}$ de las proteínas \\
\hline 1332 & Vibración en el plano $=\mathrm{CH}$ de los lípidos \\
\hline 1000 & Estiramiento del anillo de la difenilalanina \\
\hline 731 & Estiramiento y deformación del anillo de la glucosa \\
\hline
\end{tabular}

Fuente: Los autores 
Fig. 1. PRINCIPIO DE FUNCIONAMIENTO BÁSICO DE LAS PLATAFORMAS NANOESTRUCTURADAS DE PLATA; A) PROCESO DE DEPOSICIÓN Y EVAPORACIÓN DE LA MUESTRA Y SU RESPECTIVO ESPECTRO SERS; B) IMAGEN DE MICROSCOPIA DE BARRIDO ELECTRÓNICO (SEM) DE LA MUESTRA ANTES Y DESPUÉS DE SER DEPOSITADA EN LA AgNP; C) ESQUEMA DE LA INTENSIFICACIÓN DE LA SEÑAL MEDIANTE LA FORMACIÓN DE HOT SPOTS

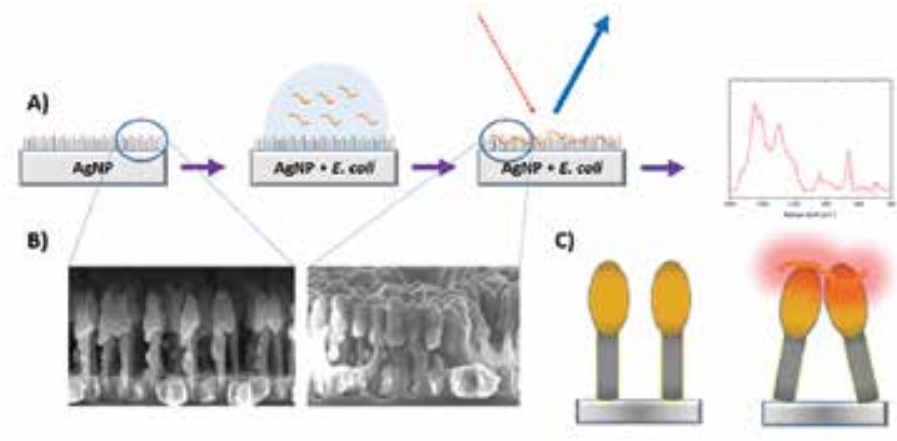

Fuente: Los autores

Fig. 2. ESPECTRO SERS DEL ÁCIDO FÓLICO SOBRE LA SUPERFICIE DE AgNPS

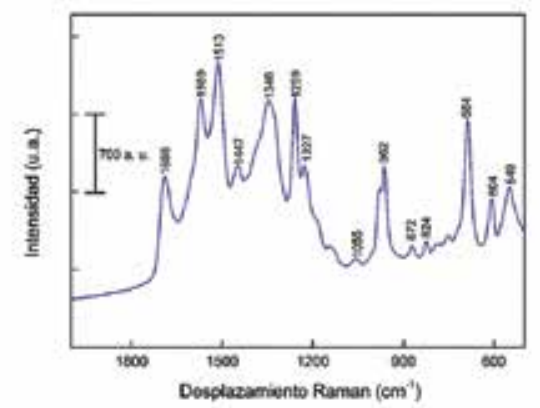

Fuente: Los autores

Fig. 3. A) ESPECTRO SERS DE LA E. COLI; B) ESPECTRO SERS DE LAS AgNP EN AUSENCIA DE E. COLI; C) INTENSIDAD DE LA SEÑAL RAMAN A 731 cm ${ }^{-1}$ DE AgNP EN PRESENCIA Y EN AUSENCIA DE E. coli; D) ESPECTRO SERS DEL MEDIO DE CULTIVO LURIA BERTINI
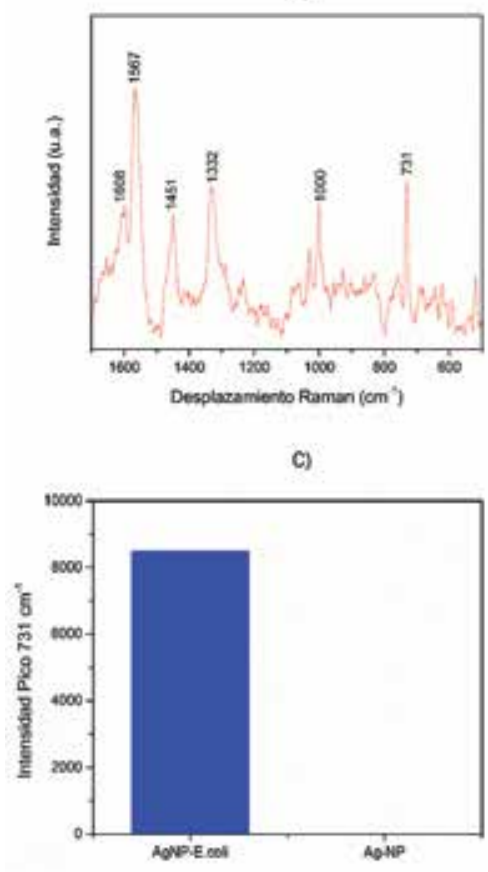

B)

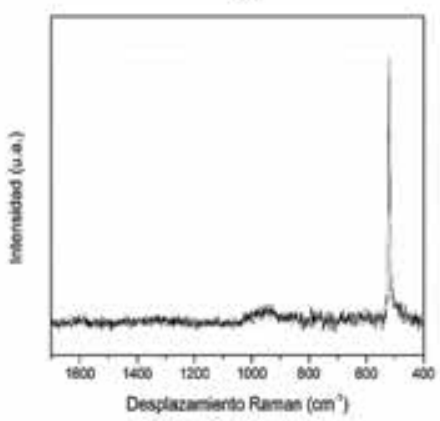

D)

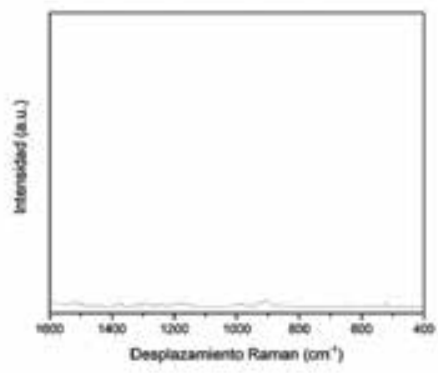

Fuente: autores. 
a un modo de vibración de tipo estiramiento del anillo de la glucosa. De esta manera las AgNP se convierten en potenciales herramientas analíticas para detección e identificación de diferentes tipos de microorganismos.

\section{AGRADECIMIENTOS}

Los autores agradecen al grupo de investigación Nanoprobes de la Universidad Técnica de Dinamarca por el soporte técnico para la fabricación de las AgNP y a la Vicerrectoría de Investigaciones de la Universidad Industrial de Santander.

\section{REFERENCIAS}

[1] M.S. Schmidt, J. Hübner, A. Boisen, “Large Area Fabrication of Leaning Silicon Nanopillars for Surface Enhanced Raman Spectroscopy", Adv Mat; 10, pp. 11-18, 2011.

[2] A.J. Driscoll, M.H. Harpster, P.A. Johnson, "The development of surface-enhanced Raman scattering as a detection modality for portable in vitro diagnostics: progress and challenges", Phys Chem Phys Chem 2013; 15, pp. 20415-20433. doi: 10.1039/c3cp52334a

[3] W.R. Premasiri, N. Krieger, W.R. Associates, "Characterization of the Surface Enhanced Raman Scattering (SERS) of Bacteria", J Chem Phys B; 109, pp. 312-320, 2005 .

[4] J. Chen, X. Wu, Y. Huang, Y. Zhao, "Chemical Detection of $E$. coli using SERS active filters with silver nanorod array", Sensors Actuators B Chem; 191, pp. 485-490, 2014

[5] J.J. Castillo, T. Rindzevicius, K. Wu et al. "Silver-capped silicon nanopillar platforms for adsorption studies of folic acid using surface enhanced Raman spectroscopy and density functional theory", J Raman Spect; 46, pp. 1087-1094, 2015.

[6] J.J. Castillo, T. Rindzevicius et al., "Adsorption and Vibrational Study of Folic Acid on Gold Nanopillar Structures Using Surface-enhanced Raman Scattering Spectroscopy Regular Paper", Nanomat Nanotechn; 5: 1-7, 2015.

[7] J. Borrero, Y. Chen, G. Dunny, Y. Kaznessis, "Modified Lactic Acid Bacteria Detect and Inhibit Multiresistant Enterococci", ACS Synthet. Biol.; 4, pp. 299-306, 2015.

[8] C. Kotanen, L. Martínez, R. Álvarez, J. Simecek, "Surface enhanced Raman scattering spectroscopy for detection and identification of microbial pathogens isolated from human serum". Sens. Biosens. Res.; 8, pp. 20-26, 2016.

[9] H. Zhou, D. Yang, N.P. Ivleva et al., "SERS Detection of Bacteria in Water by in Situ Coating with Ag Nanoparticles", Anal Chem; 86, pp. 1525-1536, 2014.

[10] D. Graham, K. Faulds, "Chemical Science Simultaneous detection and quanti fi cation of three bacterial meningitis pathogens by SERS". Chem Sci; 5, pp. 1030-1040, 2014.

[11] B. Allegranzi, Report on the Burden of Endemic Health Care-Associated Infection Worldwide Clean Care is Safer Care. WHO 2011, pp. 1-40.

[12] Secretaría de Salud de Bogotá, Sistema de vigilancia epidemiológica de infecciones intrahospitalarias. Secretaria de Salud Distrital de Bogotá, 1984; pp. 1-35

[13] K. Kneipp, H. Kneipp, B. Kartha et al., "Detection and identification of a single DNA base molecule using surface-enhanced Raman", Phys Rev E; 57, pp. 62816290, 1998.

[14] M. Culha, M. Kahraman, C. Dilek, "Rapid identification of bacteria and yeast using surface-enhanced Raman scattering", Surf Interf Anal; 42, pp. 462-465, 2010.

[15] H. Zhou, D. Yang, N. Ivleva, N. Mircescu, R. Niessner, C. Haish, "SERS Detection of Bacteria in Water by in Situ Coating with Ag Nanoparticles", Anal Chem; 86, pp. 1525-1533, 2014

[16] R.M. Jarvis, R. Goodacre, "Discrimination of Bacteria Using Surface-Enhanced Raman Spectroscopy", Anal Chem; 76, pp. 40-47, 2004.

[17] G. McNay, D. Eustace, W.E. Smith et al., "Surface-enhanced Raman scattering (SERS) and surface-enhanced resonance Raman scattering (SERRS): a review of applications", Appl Spectrosc; 65, pp. 825-37, 2011.

[18] I.S. Patel, W.R. Premasiri, D.T. Moir, L.D. Ziegler, "Barcoding bacterial cells: a SERS-based methodology for pathogen identification", J Raman Spect; 39, pp. 16601672, 2008.

[19] Y. Wang, K. Lee, J. Irudayaraj, "Silver Nanosphere SERS Probes for Sensitive Identification of Pathogens", J Phys Chem C; 114, pp. 16122-16128, 2010. 